\title{
Remembering Jane Goodman
}

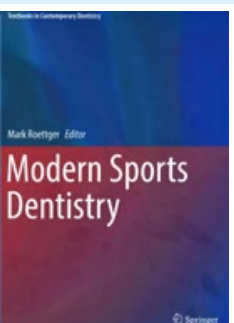

MODERN SPORTS DENTISTRY

Mark Roettger;

2018;

Springer;

price f89.99; pp 235;

ISBN: 9783319444147

Oral and maxillofacial trauma is a common risk for patients who partake in sports. Modern sports dentistry aims to provide a comprehensive overview of all aspects of trauma management and explores specific factors to consider when treating athletes. With an increasing focus on safety within sport, it appeared to be an interesting topic for the authors to review. The book is written by a large number of contributors from across multiple specialties in primary and secondary care. I feel the book is aimed at dentists who work within the medical team of a sporting club or general dental practitioners who want to update their knowledge on trauma management.

The book is divided into fourteen chapters and begins with a review of dental trauma, providing a detailed section on the diagnosis and management of such conditions. The book expands on this in subsequent chapters covering the importance restorative specialties and endodontics play in long-term dental trauma management. Prevention of dental trauma plays a central role within the book and it provides an excellent section outlining mouth guards, including the different types and methods of customisation. Through this, it provides a helpful guide for dentists in communicating their mouth guard prescriptions to their dental lab.

Maxillofacial trauma is covered in chapter five and provides an important review of hard and soft tissue injuries, alongside other trauma-related signs/symptoms which may indicate more serious underlying pathology such as head injuries. There is a chapter dedicated to dental erosion in athletes which has recently been highlighted in the sporting community to be an important issue with regards to performance. The evidence around the causes of dental erosion is well summarised.

The book is beautifully presented and contains a large number of clinical photos and radiographs which aid in the delivery of the material. There is a strong evidence-based approach to the book with scientific papers referenced throughout each chapter.

Overall, the book feels more relevant to a North American audience as it covers sports more common in that region such as American football and baseball. Many of the dental guidelines referenced are also based within the United States. The topic of sports and dentistry is niche and many of the subjects covered within the book could be found in existing paediatric, restorative or maxillofacial surgery books.

Neil Horisk 